\title{
Novel Chroman Analogs as Promising Heterocyclic Compounds: Their Synthesis and Antiepileptic Activity
}

\author{
Pinki Rawat ${ }^{1, *}$, Saurabh Manaswita Verma ${ }^{1}$, Piyush Kumar $^{2}$ \\ ${ }^{1}$ Department of Pharmaceutical Sciences and Technology, Birla Institute of Technology, Ranchi, Jharkhand, INDIA. \\ ${ }^{2}$ Faculty of Pharmacy, Integral University, Lucknow, Uttar Pradesh, INDIA.
}

\begin{abstract}
Aim: New series of novel chroman analogs was designed and synthesized using appropriate synthetic route. Materials and Methods: Structures of synthesized chroman hydrazides fused with different anhydrides were supported by spectral data. After the neurotoxicity, assessed by rotarod motor impairment method, antiepileptic activities of twenty synthesized compounds were evaluated by both Pentylenetetrazole Seizure (PTZ) and Maximal electroshock seizure (MES) methods on mice. Administration at the suitable dose level of $30 \mathrm{mg} / \mathrm{kg}, 100 \mathrm{mg} / \mathrm{kg}$ and $300 \mathrm{mg} / \mathrm{kg}$ body weight of compounds and standards was done for PTZ and MES methods and for neurotoxicity. Results: Compound $5 \mathrm{j}(30 \mathrm{mg} / \mathrm{kg})$ showed highest and advanced antiepileptic activity than reference drugs. None of the compounds showed neurotoxicity at $30 \mathrm{mg} / \mathrm{kg}$ and $100 \mathrm{mg} / \mathrm{kg}$, as determined by the rotarod test. Whereas compounds $5 \mathrm{~m}$ and $5 \mathrm{p}$ exhibited neurotoxicity at higher dose of $300 \mathrm{mg} / \mathrm{kg}$ after $4 \mathrm{hr}$. Conclusion: The results of the present study prove that the compounds have significant antiepileptic potential and are suitable candidates for further exploration.
\end{abstract}

Key words: Anhydride, Antiepileptic, Chroman, Neurotoxicity, Synthesis.

\section{INTRODUCTION}

A large section of the population around 45-100 million people is affected globally with epilepsy. ${ }^{1,2}$ It is a common neurological disorder associated with the tendency to have repeated seizures and convulsions. A seizure is characterized by a disturbance in the electrical activity of the brain. Convulsion is a symptom of an epileptic seizure associated with contraction and relaxation of body muscles rapidly and repeatedly. It can start at any age but, generally young people are more affected than old age people. ${ }^{3}$ More than 40 different Antiepileptic Drugs (AEDs) are available in clinical use, but in about one-third of patients these drugs fail to provide adequate control of epileptic seizures. ${ }^{4}$ However, dose related neurotoxicity and distinctive adverse effects limit their clinical use. ${ }^{5,6}$ About $30-40 \%$ patients are resistant to current epileptic pharmacotherapy despite availability of many different AEDs and despite understanding epileptic seizure pathogenesis. ${ }^{7}$

The different chemical classes of available AEDs include barbiturates, carbamates, hydantoin, carboxamides, succinimides, etc. The SAR of active drugs like Phenobarbital (2), Promidone, Phenytoin (3), Ethosuximide (4), Felbamate (5), Carbamazepine (6) and Clonazepam, clearly reveals the presence of amide as well as anhydride group in their structures. ${ }^{8,9}$ Some newer antiepileptic drugs which are currently under clinical trial eg, Oxcarbazine (7), Brivaracetam, Seletracetamand (1-(4-phenylpiperazin-1-yl)-3-(2(trifluoromethyl) phenyl) pyrrolidine-2, 5-dione, 8) have shown to possess amide group. ${ }^{10}$
Submission Date: 21-05-2019; Revision Date: 04-09-2019; Accepted Date: 06-10-2019

DOI: 10.5530/ijper.53.4s.162 Correspondence:

Dr. Pinki Rawat,

Department of Pharmaceutical Sciences and Technology, Birla Institute of Technology, Mesra, Ranchi-835215, Jharkhand, INDIA. Phone: +919454855617

E-mail:pnkrawat@yahoo.co.in

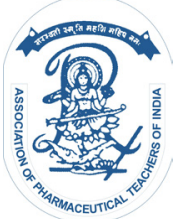

www.ijper.org 


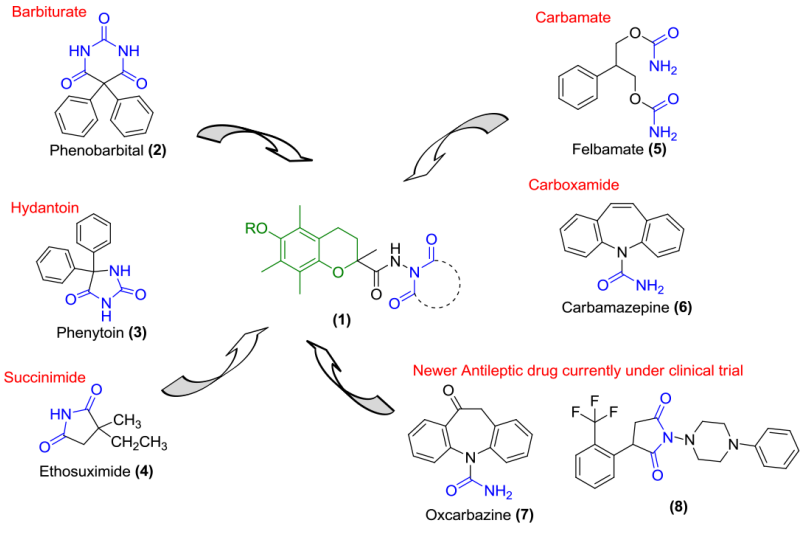

Figure 1: Pharmacophoric pattern of antiepileptic drugs and model compound.

Benzopyrans including chromans and flavonoids, due to their physico-chemical properties, have wide ranging biological applications. Review of literature reveals several pharmacological activities of chroman derivatives including anticancer, ${ }^{11,12}$ insulin release process inhibitor, ${ }^{13}$ human rhinovirus (HRV) capsid-binding inhibitor, ${ }^{14}$ anti-HIV, ${ }^{15}$ antimicrobial, ${ }^{16}$ etc. Considering the antiepileptic potential of chroman and its derivatives, the design of the chroman analogs based on AEDs pharmacophoric pattern is presented in Figure 1.

In the present study, we report the synthesis, characterization of twenty new chroman anhydride derivatives (5a-t) and report their antiepileptic activity by PTZ and MES methods along with their neurotoxicity by rotarod test.

\section{RESULTS AND DISCUSSION}

\section{Chemistry}

Synthetic strategies adopted for the synthesis of the target compounds (5a-t) described in this study has been depicted in Scheme1. Trolox (3a), conveniently synthesized by known procedure ${ }^{17}$ undergoes methylation $^{18}$ and benzylation ${ }^{19}$ to give $3 \mathrm{~b}$ and $3 \mathrm{c}$, respectively. The ester group of carboxylate present in $3 a, 3 b$ and $3 \mathrm{c}$ undergoes nucleophilie attack by hydrazine moiety resulting in $4 \mathrm{a}, 4 \mathrm{~b}$ and $4 \mathrm{c}$, respectively. ${ }^{20}$ Conversion of chroman hydracids (4a-c) to the corresponding chromananhydride derivatives $(5 \mathrm{a}-\mathrm{t})$ by condensation reaction with different anhydrides has been carried out. The properties of synthesized compounds (5a-t) are shown in Table 1.

The structures of all the newly synthesized compounds were confirmed on the basis of melting point analysis, FT-IR, ${ }^{1} \mathrm{H}-\mathrm{NMR},{ }^{13} \mathrm{C}-\mathrm{NMR}$ and Mass spectroscopy. The obtained data were in consistency with the proposed structures.

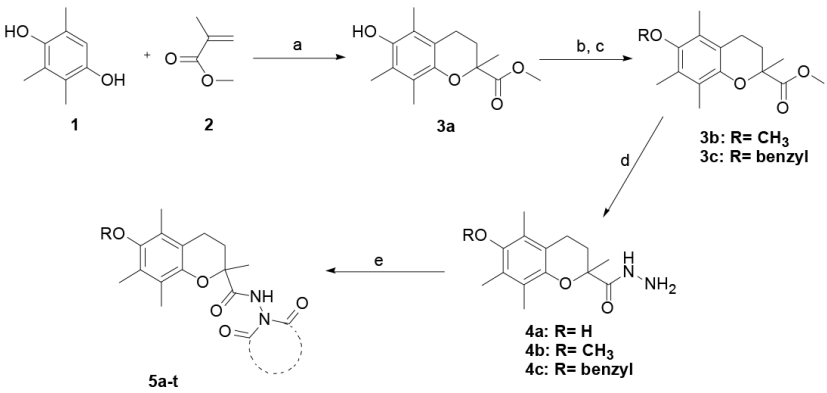

Scheme 1: Synthetic pathway for compounds 5a-t. (a) ( $\mathrm{HCHO})$ n, [ $\left.\mathrm{CH}_{3}\left(\mathrm{CH}_{2}\right)_{3}\right]_{2} \mathrm{NH}, \mathrm{CH}_{3} \mathrm{COOH}$, reflux, $20 \mathrm{hr}$; (b) dimethyl sulfate, $\mathrm{K}_{2} \mathrm{CO}_{3}, \mathrm{CH}_{3} \mathrm{COCH}_{3}, 50^{\circ} \mathrm{C}, 24 \mathrm{hr}$ (3b); (c) benzyl bromide, DMF, $\mathrm{K}_{2} \mathrm{CO}_{3}, \mathrm{RT}, 12 \mathrm{hr}$ (3c); (d) $\mathrm{NH}_{2} \mathrm{NH}_{2} \cdot \mathrm{H}_{2} \mathrm{O}, \mathrm{C}_{2} \mathrm{H}_{5} \mathrm{OH}$, reflux, $10 \mathrm{hr}$ (e) Different anhydrides, $\mathrm{CH}_{3} \mathrm{COOH}$, reflux, 2-4 hr.

The final products (5a-t) were identified by its IR spectra which showed characteristic strong absorption band at 1685-1757 of amide (-CO, stretch). Compounds 5a-i displayed a broad absorption band at 3349-3500 of hydroxy group (-OH, stretch). In ${ }^{1} \mathrm{H} \mathrm{NMR}$, the amide group $\left(-\mathrm{CONH}_{2}\right)$ was observed as a singlet at $\delta$ 8.08-10.44 ppm which was further confirmed by the disappearance of this sharp absorption peak in $\mathrm{D}_{2} \mathrm{O}$ exchange spectrum. The hydroxy group $(-\mathrm{OH})$ in compounds $(5 \mathrm{a}-\mathrm{i})$ was confirmed by the sharp singlet at around $\delta 7.49-7.51 \mathrm{ppm}$ except in compounds $5 \mathrm{~d}, 5 \mathrm{~g}$ and $5 \mathrm{i}$ (singlet at $\delta 4.32-4.39 \mathrm{ppm}$ ) due to $\mathrm{CDCl}_{3}$. The presence of singlet peak at $\delta 3.49-3.61 \mathrm{ppm}$ due to a methoxy group $\left(-\mathrm{OCH}_{3}\right)$ of $5 \mathrm{j}$-n clearly indicated methylation. A singlet peak at $\delta 4.60-4.70 \mathrm{ppm}$ was assigned to benzylic methyl $\left(-\mathrm{CH}_{2}-\right.$ )in compounds $5 \mathrm{o}-\mathrm{t}$. All of the other aromatic and aliphatic protons were present at their distinct place. In ${ }^{13} \mathrm{C}$ NMR, the signal due to carbonyl group of amide $(\mathrm{C}=\mathrm{O})$ appeared at $\delta 160.3$ $173.6 \mathrm{ppm}$. Other signals were found corresponding to their established structures. The mass spectra of almost all compounds showed $(\mathrm{M}+\mathrm{H})^{+}(\mathrm{m} / \mathrm{z})$ peaks according to their molecular weight. However, in some cases, $\left(\mathrm{M}+\mathrm{Na}^{+}\right)^{+}$and $(\mathrm{M}-\mathrm{H})^{+}(\mathrm{m} / \mathrm{z})$ peaks were also observed. Complete details are provided in the Experimental section.

\section{Antiepileptic activity}

All twenty compounds were evaluated for antiepileptic and neurological toxicity studies (e.g., ataxia, sedation, hyper-excitability) in mice using rotarod test at the doses of $30 \mathrm{mg} / \mathrm{kg}, 100 \mathrm{mg} / \mathrm{kg}$ and $300 \mathrm{mg} / \mathrm{kg}^{21}$ Data relative to the neurological toxicity (NT) and antiepileptic properties of synthesized compounds (5a-t) are shown in Table 2.

All compounds (5a-t) were devoid of toxicity upon rotarod neurotoxicity screening at the dose of $30 \mathrm{mg} / \mathrm{kg}$ 


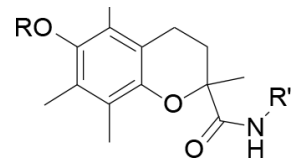

\begin{tabular}{|c|c|c|c|c|c|c|c|c|}
\hline Compd..$^{a, b}$ & Entry & $\mathbf{R}^{\prime}$ & $\begin{array}{l}\text { ReactionTime } \\
\text { (hours) }\end{array}$ & Mol. formula & Mol. weight & Yield (\%) & $\mathrm{MP}\left({ }^{\circ} \mathrm{C}\right)$ & $\mathbf{R}_{f}^{d}$ \\
\hline $5 a$ & $4 a$ & & 2 & $\mathrm{C}_{22} \mathrm{H}_{22} \mathrm{~N}_{2} \mathrm{O}_{5}$ & 394.42 & 91 & 230 & 0.6 \\
\hline $5 b$ & $4 a$ & & 2 & $\mathrm{C}_{22} \mathrm{H}_{18} \mathrm{Cl}_{4} \mathrm{~N}_{2} \mathrm{O}_{5}$ & 532.20 & 90 & 283 & 0.5 \\
\hline $5 c$ & $4 a$ & & 2 & $\mathrm{C}_{22} \mathrm{H}_{18} \mathrm{Br}_{4} \mathrm{~N}_{2} \mathrm{O}_{5}$ & 710.00 & 91 & 270 & 0.5 \\
\hline $5 d$ & $4 a$ & & 2 & $\mathrm{C}_{22} \mathrm{H}_{21} \mathrm{FN}_{2} \mathrm{O}_{5}$ & 412.41 & 68 & 218 & 0.4 \\
\hline $5 e$ & $4 a$ & & 4 & $\mathrm{C}_{21} \mathrm{H}_{21} \mathrm{~N}_{3} \mathrm{O}_{5}$ & 395.41 & 70 & 220 & 0.6 \\
\hline $5 f$ & $4 a$ & & 4 & $\mathrm{C}_{26} \mathrm{H}_{23} \mathrm{~N}_{3} \mathrm{O}_{7}$ & 489.48 & 72 & 140 & 0.7 \\
\hline $5 g$ & $4 a$ & & 2 & $\mathrm{C}_{18} \mathrm{H}_{22} \mathrm{~N}_{2} \mathrm{O}_{5}$ & 346.38 & 85 & 236 & 0.6 \\
\hline $5 \mathrm{~h}$ & $4 a$ & & 3 & $\mathrm{C}_{19} \mathrm{H}_{22} \mathrm{~N}_{2} \mathrm{O}_{5}$ & 358.39 & 86 & 218 & 0.5 \\
\hline $5 i$ & $4 a$ & & 3 & $\mathrm{C}_{18} \mathrm{H}_{18} \mathrm{Cl}_{2} \mathrm{~N}_{2} \mathrm{O}_{5}$ & 413.25 & 71 & 170 & 0.4 \\
\hline $5 j$ & $4 b$ & & 2 & $\mathrm{C}_{23} \mathrm{H}_{24} \mathrm{~N}_{2} \mathrm{O}_{5}$ & 408.45 & 88 & 210 & 0.6 \\
\hline $5 k$ & $4 b$ & & 2 & $\mathrm{C}_{23} \mathrm{H}_{20} \mathrm{Cl}_{4} \mathrm{~N}_{2} \mathrm{O}_{5}$ & 546.22 & 81 & 228 & 0.4 \\
\hline 51 & $4 b$ & & 2 & $\mathrm{C}_{23} \mathrm{H}_{20} \mathrm{Br}_{4} \mathrm{~N}_{2} \mathrm{O}_{5}$ & 724.03 & 80 & 258 & 0.5 \\
\hline
\end{tabular}

Continued... 


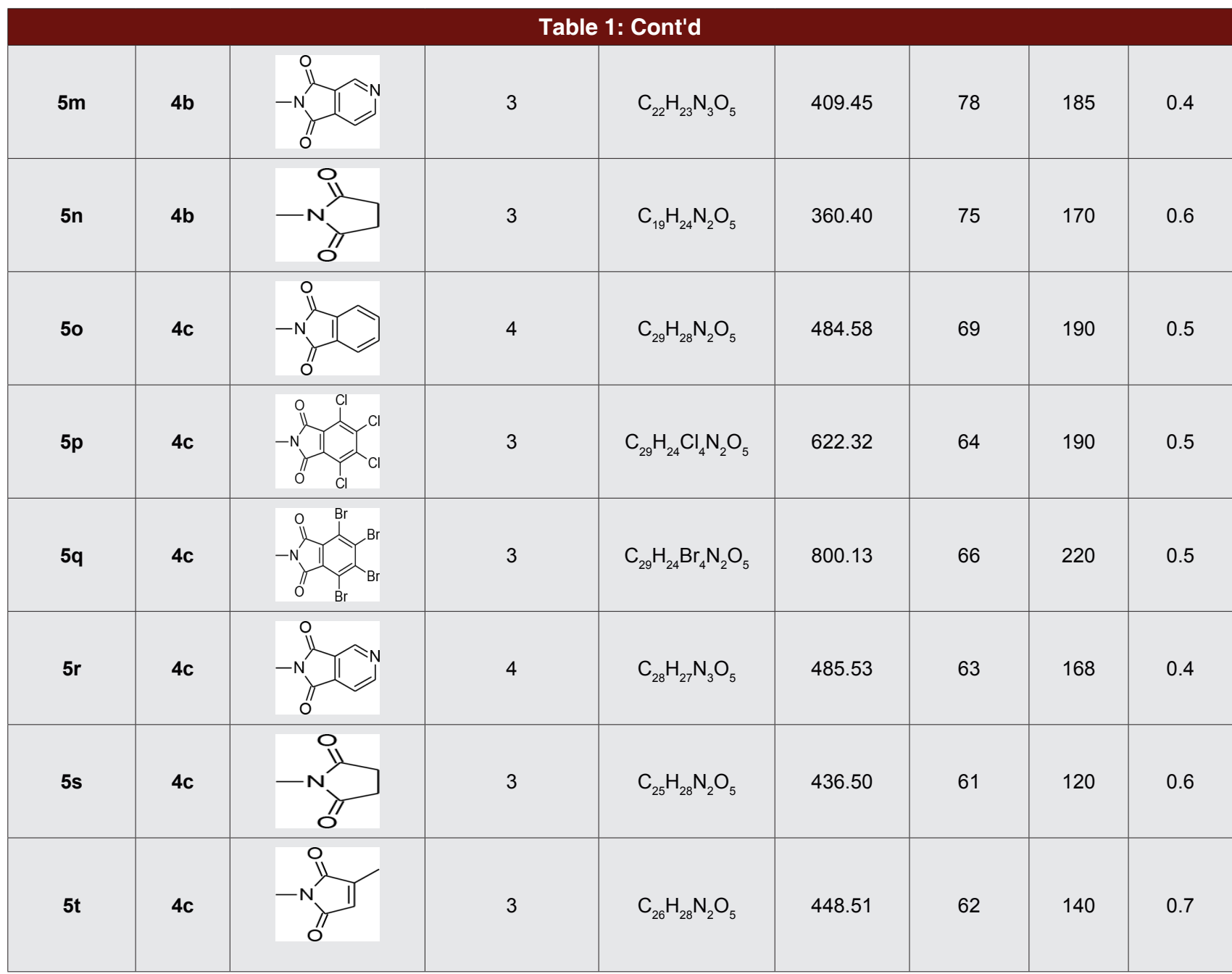

a Reagents and conditions: $5 \mathrm{a}-\mathrm{q}(0.001 \mathrm{~mol}), 4 \mathrm{a}-\mathrm{c}(0.001 \mathrm{~mol})$, acetic acid (10 ml)

${ }^{b}$ Ratio of the product was determined by the analysis of crude FT-IR, ${ }^{1} \mathrm{H}$ NMR, ${ }^{13} \mathrm{C}$ NMR and Mass spectra

'Yield refers to pure products after chromatography.

${ }^{\mathrm{d}} \mathrm{R} f=\mathrm{EtOAC/Hexane}(40: 60)$

and $100 \mathrm{mg} / \mathrm{kg}$ at $0.5 \mathrm{hr}$. While as compounds $5 \mathrm{~m}$ and $5 \mathrm{p}$ were toxic at the dose of $300 \mathrm{mg} / \mathrm{kg}$ after $4 \mathrm{~h}$. However, all the compounds were less toxic than standard drug, phenytoin $(100 \mathrm{mg} / \mathrm{kg})$.

The results of antiepileptic study of compounds (5a-t) by PTZ method showed that all compounds displayed significant activity. Most active compounds $5 \mathrm{e}, 5 \mathrm{f}$ and $5 \mathrm{hr}$ presented protection at the dose level of $30 \mathrm{mg} /$ $\mathrm{kg}$ after both the time intervals $0.5 \mathrm{~h}$ and $4 \mathrm{hr}$. Whereas compounds $5 \mathrm{a}, 5 \mathrm{~b}, 5 \mathrm{j}, 5 \mathrm{k}$ and $5 \mathrm{p}$ showed protection at $100 \mathrm{mg} / \mathrm{kg}$ dose level after $4 \mathrm{hr}$. Compounds 5c, 5d, $5 \mathrm{i}, 5 \mathrm{l}, 5 \mathrm{~m}, 5 \mathrm{o}$ and $5 \mathrm{~s}$ exhibited protection at $100 \mathrm{mg} / \mathrm{kg}$ dose level after $0.5 \mathrm{~h}$ and $4 \mathrm{hr}$ time intervals while compounds $5 \mathrm{n}, 5 \mathrm{q}$ and $5 \mathrm{r}$ showed protection at 300 $\mathrm{mg} / \mathrm{kg}$ dose level after $4 \mathrm{hr}$. The remaining compounds (5g and 5t) had activity at the dose level of $300 \mathrm{mg} / \mathrm{kg}$ at both the time intervals. Compound $5 \mathrm{f}$ showed good activity which may be due to increase in the ring size as other compounds $(5 \mathrm{~g}, 5 \mathrm{i}, 5 \mathrm{n}$ and $5 \mathrm{~s}$ ) having smaller ring system possessed less activity. It seemed that substitution of $6-\mathrm{OH}$ group resulted in more active compounds (5a, 5b, 5e, 5f and 5hr). Amongst the electronegative groups (chloro, bromo and fluoro) in tested compounds, chloro derivatives (5b, $5 \mathrm{k}$ and $5 \mathrm{p}$ ) showed better activity.

All compounds (5a-t) displayed protection in PTZ model (Table 2). Therefore compounds may have inhibited or attained PTZ-induced seizure in mice by increasing GABAergic neurotransmission. ${ }^{22}$

Maximal Electroshock Seizure (MES) method when applied on mice showed reasonable activity by several compounds. Most potent compounds $5 j$ and $5 t$ were comparable in activity with result to standard drug, phenytoin at dose level of $30 \mathrm{mg} / \mathrm{kg}$ after the time periods of $0.5 \mathrm{~h}$ and $4 \mathrm{~h}$. Furthermore, compounds $5 \mathrm{f}$, 


\section{Table 2: Effect of compounds (5a-t) administered orally at the dose of $30 \mathrm{mg} / \mathrm{kg}, 100 \mathrm{mg} / \mathrm{kg}$ and $300 \mathrm{mg} / \mathrm{kg}$ on} convulsions induced by PTZ and MES andon neurotoxicity (NT).

\begin{tabular}{|c|c|c|c|c|c|c|}
\hline \multirow[t]{3}{*}{ Compounds } & \multicolumn{6}{|c|}{ Intraperitoneal injection in mice $(\mathrm{mg} / \mathrm{kg})$} \\
\hline & \multicolumn{2}{|c|}{ NT } & \multicolumn{2}{|c|}{ PTZ } & \multicolumn{2}{|c|}{ MES } \\
\hline & $0.5 \mathrm{hr}$ & $4 \mathrm{hr}$ & $0.5 \mathrm{~h}$ & $4 \mathrm{hr}$ & $0.5 \mathrm{hr}$ & $4 \mathrm{hr}$ \\
\hline $5 a$ & - & - & 30 & 100 & - & - \\
\hline $5 b$ & - & - & 30 & 100 & - & - \\
\hline $5 c$ & - & - & 100 & 100 & - & - \\
\hline $5 d$ & - & - & 100 & 100 & - & - \\
\hline $5 e$ & - & - & 30 & 30 & 300 & - \\
\hline $5 f$ & - & - & 30 & 30 & 100 & - \\
\hline $5 \mathrm{~g}$ & - & - & 300 & 300 & - & - \\
\hline $5 \mathrm{~h}$ & - & - & 30 & 30 & - & - \\
\hline $5 i$ & - & - & 100 & 100 & 100 & 100 \\
\hline $5 j$ & - & - & 30 & 100 & 30 & 30 \\
\hline $5 k$ & - & - & 30 & 100 & - & 300 \\
\hline $5 I$ & - & - & 100 & 100 & - & 100 \\
\hline $5 m$ & - & 300 & 100 & 100 & 300 & 300 \\
\hline $5 n$ & - & - & 100 & 300 & 100 & 100 \\
\hline 50 & - & - & 100 & 100 & 100 & 100 \\
\hline $5 p$ & - & 300 & 30 & 100 & - & 300 \\
\hline $5 q$ & - & - & 100 & 300 & - & - \\
\hline $5 r$ & - & - & 100 & 300 & 300 & - \\
\hline $5 s$ & - & - & 100 & 100 & - & - \\
\hline $5 t$ & - & - & 300 & 300 & 30 & 30 \\
\hline Phenytoin & 100 & 100 & & & 30 & 30 \\
\hline Sodium Valproate & & & 200 & 200 & & \\
\hline
\end{tabular}

${ }^{a}$ The figure in the table indicates minimum dose required for bioactivity in half or more of the mice examined at $0.5 \mathrm{hr}$ and $4 \mathrm{hr}$.

${ }^{\mathrm{b}}$ Dash indicates the absence of antiepileptic activity and neurotoxicity at the maximum dose administered (300 $\left.\mathrm{mg} / \mathrm{kg}\right)$.

$5 \mathrm{n}$ and $5 \mathrm{o}$ had protection of seizures in mice at the dose level of $100 \mathrm{mg} / \mathrm{kg}$ after both time periods except for the compound $5 \mathrm{f}$ which showed protection only after $0.5 \mathrm{hr}$. However, in case of compounds $5 \mathrm{k}, 5 \mathrm{~m}$ and $5 \mathrm{p}$ protection of seizures was observed at higher dose of $300 \mathrm{mg} / \mathrm{kg}$. It had been found that compounds with hydroxyl group at position 6 of the envisaged compounds possessed less activity in comparison to other groups (methoxy and benzyl) at the same position. The occupancy of electron withdrawing groups (chloro, bromo and fluoro) might not had significant effect on activity as compounds $5 \mathrm{~b}, 5 \mathrm{c}, 5 \mathrm{~d}, 5 \mathrm{k}, 5 \mathrm{l}, 5 \mathrm{p}$ and $5 \mathrm{q}$ were less active or inactive.

\section{MATERIALS AND METHODS}

Melting points were checked in open glass capillaries and are uncorrected. The TLC of the compound used E Merck silica gel GF-254 precoated plates (Merck, Darmstadt, Germany). NMR spectra were determined on JEOL ECX-500 spectrometer in DMSO-d6 and $\mathrm{CDCl}_{3}\left(400\right.$ and $500 \mathrm{MHz}$ ) for ${ }^{1} \mathrm{H}$ and $125 \mathrm{MHz}$ for ${ }^{13} \mathrm{C}$. The chemical shifts were recorded in $(\delta, \mathrm{ppm})$ relative to TMS as an internal standard. The mass spectra were determined by Waters-Q-T of Premier-HAB213 spectrometer and Microscopic II triple Quadrupole mass spectrometer using EI and the $m / z$ values are indicated in Dalton. Infrared spectra $(\mathrm{KBr})$ were recorded by using Bruker FT/IR Vector 22 spectrophotometer.

\section{Synthesis of 3a-c}

The compounds were prepared by a literature procedure ${ }^{11}$ which involved methylation and benzylation. The material was recrystallized from methanol and purification was done by column chromatography (pet. ether/ EtOAc 90/10).

\section{Synthesis of $4 a-c$}

The compounds were prepared by a literature procedure. ${ }^{11}$ It includes nucleophillic addition by hydrazine. 
(a) 6-Hydroxy-2,5,7,8-tetramethyl-3,4-dihydro2H-chromene-2-carbohydrazide (4a)

Yield: White solid (60\%); ${ }^{1} \mathrm{HNMR}(500 \mathrm{MHz}$, DMSO$\mathrm{d}_{6}, \quad$ ppm): $8.47(\mathrm{~s}, 1 \mathrm{H}, \mathrm{OH}), 7.43(\mathrm{~s}, 1 \mathrm{H},-\mathrm{NH}), 4.19$ $\left(\mathrm{s}, 2 \mathrm{H}, \mathrm{NH}_{2}\right), 2.52-2.49(\mathrm{~m}, 1 \mathrm{H}), 2.40-2.37(\mathrm{~m}, 1 \mathrm{H}), 2.20-$ $2.15(\mathrm{~m}, 1 \mathrm{H}), 2.04(\mathrm{~s}, 3 \mathrm{H}), 2.02(\mathrm{~s}, 3 \mathrm{H}), 1.94(\mathrm{~s}, 3 \mathrm{H}), 1.68-$ $1.63(\mathrm{~m}, 1 \mathrm{H}), 1.35(\mathrm{~s}, 3 \mathrm{H})$.

(b) 6-Methoxy-2,5,7,8-tetramethyl-3,4-dihydro2H-chromene-2-carbohydrazide (4b)

Yield: White solid (90\%); ${ }^{1} \mathrm{H}$ NMR $\left(400 \mathrm{MHz}, \mathrm{CDCl}_{3}\right.$, ppm): 7.51 (s,1H,-NH), 3.81-3.80 (d,2H,- $\left.\mathrm{NH}_{2}\right), 3.60$ $(\mathrm{s}, 3 \mathrm{H}), 2.63-2.56(\mathrm{~m}, 1 \mathrm{H}), 2.52-2.45(\mathrm{~m}, 1 \mathrm{H}), 2.37-2.31$ $(\mathrm{m}, 1 \mathrm{H}), 2.18(\mathrm{~s}, 3 \mathrm{H}), 2.12(\mathrm{~s}, 3 \mathrm{H}), 1.90-1.85(\mathrm{~m}, 1 \mathrm{H}), 1.52$ $(\mathrm{s}, 3 \mathrm{H})$.

(c) 6-(Benzyloxy)-2,5,7,8-tetramethyl-3,4-dihydro-2H-chromene-2-carbohydrazide (4c) Yield: White solid (71\%); ${ }^{1} \mathrm{H}$ NMR $\left(400 \mathrm{MHz}, \mathrm{CDCl}_{3}\right.$, ppm): 7.53 (s,1H,-NH), 7.48-7.37 (m,5H, Ph), 4.67 $(\mathrm{s}, 2 \mathrm{H}), 3.82-3.81\left(\mathrm{~d}, 2 \mathrm{H},-\mathrm{NH}_{2}\right), 2.64-2.58(\mathrm{~m}, 1 \mathrm{H}), 2.54-$ $2.46(\mathrm{~m}, 1 \mathrm{H}), 2.39-2.33(\mathrm{~m}, 1 \mathrm{H}), 2.21(\mathrm{~s}, 3 \mathrm{H}), 2.14(\mathrm{~s}, 3 \mathrm{H})$, $2.13(\mathrm{~s}, 3 \mathrm{H}), 1.91-1.87(\mathrm{~m}, 1 \mathrm{H}), 1.53(\mathrm{~s}, 3 \mathrm{H})$.

\section{General procedure for the synthesis of final compounds (5a-t)}

A mixture of selected chroman-hydrazides (4a-c, 100 $\mathrm{mmol})$ and different anhydrides $(0.1 \mathrm{~mol})$ in glacial acetic acid was refluxed for $2-4 \mathrm{hr}$ to complete the reaction and lead to final compounds $5 \mathrm{a}-\mathrm{t}$.

(a) $\quad N$-(1,3-dioxoisoindolin-2-yl)-6-hydroxy$2,5,7,8$-tetramethylchroman-2-carboxamide (5a)

Yellow solid; IR $\left(\mathrm{KBr}, \mathrm{v}, \mathrm{cm}^{-1}\right): 3379(>\mathrm{NH}), 1687$ (>CO strech); ${ }^{1} \mathrm{H}$ NMR (400 MHz, DMSO-d, $\left.{ }_{6}, \mathrm{ppm}\right)$ : 10.23 (s, -CONH-, exchangeable with $\mathrm{D}_{2} \mathrm{O}$ ), 7.94-7.88 $(\mathrm{m}, 4 \mathrm{H}), 7.49$ (s, - $\mathrm{OH}$, exchangeable with $\left.\mathrm{D}_{2} \mathrm{O}\right), 2.61$ $2.53\left(\mathrm{~m}, 2 \mathrm{H},>\mathrm{CH}_{2}\right), 2.26-2.20\left(\mathrm{~m}, 1 \mathrm{H},>\mathrm{CH}_{2}\right), 2.07$ $\left(\mathrm{s}, 3 \mathrm{H},-\mathrm{CH}_{3}\right), 2.04\left(\mathrm{~s}, 3 \mathrm{H},-\mathrm{CH}_{3}\right), 2.00\left(\mathrm{~s}, 3 \mathrm{H},-\mathrm{CH}_{3}\right)$, 1.83-1.77 (m, $\left.1 \mathrm{H},>\mathrm{CH}_{2}\right), 1.46\left(\mathrm{~s}, 3 \mathrm{H},-\mathrm{CH}_{3}\right)$; HR-MS: $395.1607(\mathrm{M}+\mathrm{H})^{+}$, calcd. 395.1609.

(b) 6-hydroxy-2,5,7,8-tetramethyl- $N-(4,5,6,7-$ tetrachloro-1,3-dioxoisoindolin-2-yl)chroman-2-carboxamide (5b)

Red solid; IR (KBr, $\left.v, \mathrm{~cm}^{-1}\right): 3473(-\mathrm{OH}), 3393(>\mathrm{NH})$, 2937 (aliphatic C-H), 1699 (>CO strech); ${ }^{1} \mathrm{H}$ NMR (400 MHz, DMSO-d, ppm): 10.44 (s, -CONH), 7.51 (s, - $\mathrm{OH}-), 2.62-2.49\left(\mathrm{~m}, 2 \mathrm{H},>\mathrm{CH}_{2}\right), 2.28-2.22(\mathrm{~m}, 1 \mathrm{H})$, $2.06\left(\mathrm{~s}, 3 \mathrm{H},-\mathrm{CH}_{3}\right), 2.03\left(\mathrm{~s}, 3 \mathrm{H},-\mathrm{CH}_{3}\right), 1.99\left(\mathrm{~s}, 3 \mathrm{H},-\mathrm{CH}_{3}\right)$, 1.82-1.75 (m, 1H), $1.47\left(\mathrm{~s}, 3 \mathrm{H},-\mathrm{CH}_{3}\right) ;{ }^{13} \mathrm{C}$ NMR $(125$ MHz, DMSO-d $): 173.4$ (-CONH-), $161.2(>\mathrm{C}=\mathrm{O}$, $>\mathrm{C}=\mathrm{O}), 146.5,144.2,139.9,129.5,126.4,123.3,121.9$, 120.6, 117.5, $77.9(>\mathrm{C}<), 30.4\left(-\mathrm{CH}_{2}\right), 24.4\left(-\mathrm{CH}_{3}\right), 20.3$
$\left(-\mathrm{CH}_{2}\right), 13.3\left(-\mathrm{CH}_{3}\right), 12.7\left(-\mathrm{CH}_{3}\right), 12.3\left(-\mathrm{CH}_{3}\right)$; HR-MS: $528.9888(\mathrm{M}+\mathrm{H})^{+}$, calcd. 528.9891.

(c) 6-hydroxy-2,5,7,8-tetramethyl- $N-(4,5,6,7-$ tetrabromo-1,3-dioxoisoindolin-2-yl)chroman-2-carboxamide $(5 \mathrm{c})$

Red solid; IR (KBr, v, cm $\left.{ }^{-1}\right): 3479(-\mathrm{OH}), 3377(>\mathrm{NH})$, 2941 (aliphatic C-H), 1699 (>CO strech); ${ }^{1} \mathrm{H}$ NMR (400 MHz, DMSO-d, ppm): 10.39 (s, -CONH), 7.50 (s, -OH-), 2.60-2.49 (m, $\left.2 \mathrm{H},>\mathrm{CH}_{2}\right), 2.28-2.22(\mathrm{~m}, 1 \mathrm{H})$, $2.06\left(\mathrm{~s}, 3 \mathrm{H},-\mathrm{CH}_{3}\right), 2.03\left(\mathrm{~s}, 3 \mathrm{H},-\mathrm{CH}_{3}\right), 1.99$ (s, 3H, $\left.-\mathrm{CH}_{3}\right), 1.82-1.75(\mathrm{~m}, 1 \mathrm{H}), 1.47\left(\mathrm{~s}, 3 \mathrm{H},-\mathrm{CH}_{3}\right) ;{ }^{13} \mathrm{C}$ NMR (125 MHz, DMSO-d 6 ): 173.4 (-CONH-), 161.6 $(>\mathrm{C}=0,>\mathrm{C}=0), 146.4,144.2,138.2,129.2,123.3,121.9$, 120.6, 117.5, $77.9(>\mathrm{C}<), 30.4\left(-\mathrm{CH}_{2}\right), 24.5\left(-\mathrm{CH}_{3}\right), 20.3$ $\left(-\mathrm{CH}_{2}\right), 13.3\left(-\mathrm{CH}_{3}\right), 12.7\left(-\mathrm{CH}_{3}\right), 12.4\left(-\mathrm{CH}_{3}\right)$; HR-MS: $704.7870(\mathrm{M}+\mathrm{H})^{+}$,calcd. 704.7880 .

(d) $\quad \mathrm{N}$-(4-fluoro-1,3-dioxoisoindolin-2-yl)-6-hydroxy-2,5,7,8-tetramethylchroman-2-carboxamide (5d)

Light green solid; IR ( $\left.\mathrm{KBr}, \mathrm{v}, \mathrm{cm}^{-1}\right): 3369$ (-OH stretch), 2926 (aliphatic C-H), 1686 (>CO stretch); ${ }^{1} \mathrm{H}$ NMR (400 MHz, $\left.\mathrm{CDCl}_{3}, \mathrm{ppm}\right): 8.17$ (s, -CONH-), 7.79-7.74 $(\mathrm{m}, 1 \mathrm{H}), 7.70(\mathrm{~d}, J=7.3 \mathrm{~Hz}, 1 \mathrm{H}), 7.42(\mathrm{t}, J=8.4 \mathrm{~Hz}, 1 \mathrm{H})$, 4.34 (s, -OH), 2.76-2.65 (m, 2H, >CH2), 2.43-2.36 (m, $1 \mathrm{H}), 2.19\left(\mathrm{~s}, 3 \mathrm{H},-\mathrm{CH}_{3}\right), 2.16\left(\mathrm{~s}, 3 \mathrm{H},-\mathrm{CH}_{3}\right), 2.12(\mathrm{~s}, 3 \mathrm{H}$, $\left.-\mathrm{CH}_{3}\right), 1.97-1.90(\mathrm{~m}, 1 \mathrm{H}), 1.63\left(\mathrm{~s}, 3 \mathrm{H},-\mathrm{CH}_{3}\right) ;{ }^{13} \mathrm{C} \mathrm{NMR}$ $\left(100 \mathrm{MHz}, \mathrm{CDCl}_{3}\right): 173.5$ (-CONH-),163.8 (>C=0), $161.5(>\mathrm{C}=0), 145.9,143.7,137.4,137.3,132.1,123.2$, 123.0, 122.1, 121.6, 120.2, 119.0, 118.1, 78.9 $(>\mathrm{C}<)$, $30.1\left(-\mathrm{CH}_{2}\right), 24.0\left(-\mathrm{CH}_{3}\right), 20.2\left(-\mathrm{CH}_{2}\right), 12.3\left(-\mathrm{CH}_{3}\right), 12.2$ $\left(-\mathrm{CH}_{3}\right), 11.4\left(-\mathrm{CH}_{3}\right)$; HR-MS: $413.1512(\mathrm{M}+\mathrm{H})^{+}$, calcd. 413.1511.

(e) $\quad N$-(1,3-dioxo-1H-pyrrolo[3,4-c]pyridin2(3H)-yl)-6-hydroxy-2,5,7,8-tetramethylchroman-2-carboxamide (5e)

Orange solid; IR $\left(\mathrm{KBr}, \mathrm{v}, \mathrm{cm}^{-1}\right)$ : 3379 (-OH stretch), 2930 (aliphatic C-H), 1715 (>CO stretch); ${ }^{1} \mathrm{H}$ NMR (400 MHz, DMSO-d ${ }_{6}$, ppm): 10.37 (s, -CONH-), 9.19$9.16(\mathrm{~m}, 2 \mathrm{H}), 7.96-7.95(\mathrm{~m}, 1 \mathrm{H}), 7.50(\mathrm{~s},-\mathrm{OH}), 2.61-2.53$ $\left(\mathrm{m}, 2 \mathrm{H},>\mathrm{CH}_{2}\right), 2.28-2.21\left(\mathrm{~m}, 1 \mathrm{H},>\mathrm{CH}_{2}\right), 2.07(\mathrm{~s}, 3 \mathrm{H}$, $\left.-\mathrm{CH}_{3}\right), 2.03\left(\mathrm{~s}, 3 \mathrm{H},-\mathrm{CH}_{3}\right), 1.99\left(\mathrm{~s}, 3 \mathrm{H},-\mathrm{CH}_{3}\right), 1.83-1.76$ (m, $1 \mathrm{H},>\mathrm{CH}_{2}$ ), 1.47 (s, 3H, $-\mathrm{CH}_{3}$ ); HR-MS: 396.1559 $(\mathrm{M}+\mathrm{H})^{+}$, calcd. 396.1559 .

(f) 6-hydroxy-2,5,7,8-tetramethyl-N-(5-nitro1,3-dioxo-1 $H$-benzo[de]isoquinolin-2(3H)yl)chroman-2-carboxamide (5f)

Orange solid; IR ( $\left.\mathrm{KBr}, v, \mathrm{~cm}^{-1}\right): 3403$ (-OH stretch), 2926 (aliphatic C-H), 1698 (>CO stretch); ${ }^{1} \mathrm{H}$ NMR (400 MHz, DMSO-d, ppm): 10.34 (s, -CONH), 9.52(s, $1 \mathrm{H}), 8.97\left(\mathrm{~d}, J=12.8 \mathrm{H}_{2}, 1 \mathrm{H}\right), 8.82(\mathrm{~d}, J=8.2 \mathrm{~Hz}, 1 \mathrm{H})$, $8.70(\mathrm{dd}, J=7.4 \mathrm{~Hz}, J=12.2 \mathrm{~Hz}, 1 \mathrm{H}), 8.06$ (dd, J=7.4 Hz, 
$J=13.7 \mathrm{~Hz}, 1 \mathrm{H}), 7.51$ (s, -OH-), 2.63-2.61 (m, 2H), 2.36$2.30(\mathrm{~m}, 1 \mathrm{H}), 2.09$ (s, 3H, $\left.-\mathrm{CH}_{3}\right), 2.04\left(\mathrm{~s}, 3 \mathrm{H},-\mathrm{CH}_{3}\right)$, $2.02\left(\mathrm{~s}, 3 \mathrm{H},-\mathrm{CH}_{3}\right), 1.86-1.80(\mathrm{~s}, 1 \mathrm{H}), 1.52\left(\mathrm{~s}, 3 \mathrm{H},-\mathrm{CH}_{3}\right)$; ${ }^{13} \mathrm{C}$ NMR (100 MHz, DMSO-d $)$ : 172.9 (-CONH-), $161.3(>\mathrm{C}=0), 161.2(>\mathrm{C}=0), 146.5,146.4,146.4,137.8$, 135.3, 131.7, 131.2, 129.9, 129.7, 124.5, 123.7, 123.2, $122.3,121.8,120.6,117.7,78.1(>\mathrm{C}<), 30.5\left(-\mathrm{CH}_{2}\right)$, $24.4\left(-\mathrm{CH}_{3}\right), 20.3\left(-\mathrm{CH}_{2}\right), 13.3\left(-\mathrm{CH}_{3}\right), 12.7\left(-\mathrm{CH}_{3}\right), 12.4$ $\left(-\mathrm{CH}_{3}\right)$; HR-MS: $512.1433(\mathrm{M}+\mathrm{Na})^{+}$,calcd. 512.1439.

(g) $\quad N$-(2,5-dioxopyrrolidin-1-yl)-6-hydroxy2,5,7,8-tetramethylchroman-2-carboxamide (5g)

White solid; IR (KBr, v, cm $\left.{ }^{-1}\right): 3500$ (-OH stretch), 2933 (aliphatic C-H), 1707 (>CO stretch); ${ }^{1} \mathrm{H}$ NMR (400 MHz, $\mathrm{CDCl}_{3}, \mathrm{ppm}$ ): 8.08 (s, -CONH-), 4.39 (s, 1H, $-\mathrm{OH}), 2.78\left(\mathrm{~s}, 4 \mathrm{H},>\mathrm{CH}_{2}\right), 2.70-2.65\left(\mathrm{~m}, 2 \mathrm{H},>\mathrm{CH}_{2}\right)$, 2.38-2.30 (m, $\left.1 \mathrm{H},>\mathrm{CH}_{2}\right), 2.14\left(\mathrm{~s}, 6 \mathrm{H},-\mathrm{CH}_{3}\right), 2.10(\mathrm{~s}, 3 \mathrm{H}$, $\left.-\mathrm{CH}_{3}\right), 1.59$ (s, 3H, $\left.-\mathrm{CH}_{3}\right)$; HR-MS: $347.1600(\mathrm{M}+\mathrm{H})^{+}$, calcd. 347.1600 .

(h) $6-$ hydroxy $-2,5,7,8-\operatorname{tet}$ a methy $1-N$ (3-methyl-2,5-dioxo-2,5-dihydro- $1 H$-pyrrol1-yl)chroman-2-carboxamide (5h)

Yellowish-orange solid; IR (KBr, v, $\left.\mathrm{cm}^{-1}\right)$ : 3349 (-OH stretch), 2923 (aliphatic C-H), 1685 (>CO stretch); ${ }^{1} \mathrm{H}$ NMR (400 MHz, DMSO-d,, ppm): 10.01 (s, -CONH), 7.50 (s, -OH-), 6.75 (d, J=1.8 H₹, 1H), 2.56-2.43 $\left(\mathrm{m}, 2 \mathrm{H},>\mathrm{CH}_{2}\right), 2.20-2.14(\mathrm{~m}, 1 \mathrm{H}), 2.04\left(\mathrm{~s}, 3 \mathrm{H},-\mathrm{CH}_{3}\right)$, 2.02 (s, 3H, - $\left.\mathrm{CH}_{3}\right), 1.99$ (d, 3H, - $\left.\mathrm{CH}_{3}\right), 1.97$ (s, 3H, $\left.-\mathrm{CH}_{3}\right), 1.78-1.71(\mathrm{~m}, 1 \mathrm{H}), 1.40\left(\mathrm{~s}, 3 \mathrm{H},-\mathrm{CH}_{3}\right) ;{ }^{13} \mathrm{C} \mathrm{NMR}$ (100 MHz, DMSO-d ): 173.5 (-CONH-), $169.4(>\mathrm{C}=0)$, $168.3(>\mathrm{C}=0), 146.4,145.3,144.2(>\mathrm{C}<), 127.3(>\mathrm{C}<)$, $123.2,121.9,120.6,117.5,77.7(>\mathrm{C}<), 30.4\left(-\mathrm{CH}_{2}\right)$, $24.3\left(-\mathrm{CH}_{3}\right), 20.2\left(-\mathrm{CH}_{2}\right), 13.2\left(-\mathrm{CH}_{3}\right), 12.7\left(-\mathrm{CH}_{3}\right), 12.3$ $\left(-\mathrm{CH}_{3}\right), 11.5\left(-\mathrm{CH}_{3}\right)$; HR-MS: $381.1421(\mathrm{M}+\mathrm{Na})^{+}$, calcd. 381.1420 .

(i) $\quad \mathrm{N}$-(3,4-dichloro-2,5-dioxo-2,5-dihydro$1 H$-pyrrol-1-yl)-6-hydroxy-2,5,7,8-tetramethylchroman-2-carboxamide (5i)

Brown solid; IR $\left(\mathrm{KBr}, \mathrm{v}, \mathrm{cm}^{-1}\right)$ : 3382 (-OH stretch), 2929 (aliphatic C-H), 1757 (>CO stretch); ${ }^{1} \mathrm{H}$ NMR (400 $\left.\mathrm{MHz}, \mathrm{CDCl}_{3}, \mathrm{ppm}\right): 8.07$ (s, -CONH-), 4.32 $(\mathrm{s},-\mathrm{OH}), 2.66\left(\mathrm{t}, \mathrm{J}=6.4 \mathrm{~Hz}, 2 \mathrm{H},>\mathrm{CH}_{2}\right), 2.39-2.32$ $\left(\mathrm{m}, 1 \mathrm{H},>\mathrm{CH}_{2}\right), 2.17\left(\mathrm{~s}, 3 \mathrm{H},-\mathrm{CH}_{3}\right), 2.15\left(\mathrm{~s}, 3 \mathrm{H},-\mathrm{CH}_{3}\right)$, $2.11\left(\mathrm{~s}, 3 \mathrm{H},-\mathrm{CH}_{3}\right), 1.95-1.88\left(\mathrm{~m}, 1 \mathrm{H},>\mathrm{CH}_{2}\right), 1.59(\mathrm{~s}, 3 \mathrm{H}$, $\left.{ }^{-\mathrm{CH}_{3}}\right) ;{ }^{13} \mathrm{C} \mathrm{NMR}\left(100 \mathrm{MHz}, \mathrm{CDCl}_{3}\right)$ : $173.6(>\mathrm{C}=\mathrm{O})$, $160.3(>\mathrm{C}=\mathrm{O}), 146.0,143.6,133.2,122.1,121.7,119.1$, 118.0, $78.8(>\mathrm{C}<), 30.0\left(-\mathrm{CH}_{2}\right), 23.9\left(-\mathrm{CH}_{3}\right), 20.1\left(-\mathrm{CH}_{2}\right)$, 14.2, 12.3, 12.2, 11.3; HR-MS: $413.0671(\mathrm{M}+\mathrm{H})^{+}$, calcd. 413.0656 .
$N$-(1,3-dioxoisoindolin-2-y1)-6-methoxy$2,5,7,8$-tetramethylchroman-2-carboxamide (5j)

White solid; IR ( $\left.\mathrm{KBr}, \mathrm{v}, \mathrm{cm}^{-1}\right): 3409$ (>NH stretch), 2932 (aliphatic C-H), 1707 (>CO stretch); ${ }^{1} \mathrm{H}$ NMR (400 MHz, DMSO-d,, ppm): 10.31 (s, -CONH-), 7.94$7.88(\mathrm{~m}, 4 \mathrm{H}), 3.50\left(\mathrm{~s}, 3 \mathrm{H},-\mathrm{OCH}_{3}\right), 2.63-2.54(\mathrm{~m}, 2 \mathrm{H}$, $\left.>\mathrm{CH}_{2}\right), 2.30-2.24\left(\mathrm{~m}, 1 \mathrm{H},>\mathrm{CH}_{2}\right), 2.08\left(\mathrm{~s}, 3 \mathrm{H},-\mathrm{CH}_{3}\right)$, $2.07\left(\mathrm{~s}, 3 \mathrm{H},-\mathrm{CH}_{3}\right), 2.04\left(\mathrm{~s}, 3 \mathrm{H},-\mathrm{CH}_{3}\right), 1.84-1.77(\mathrm{~m}, 1 \mathrm{H}$, $\left.>\mathrm{CH}_{2}\right), 1.49$ (s, 3H, $-\mathrm{CH}_{3}$ ); HR-MS: $409.1763(\mathrm{M}+\mathrm{H})^{+}$, calcd. 409.1761.

(k) 6-methoxy-2,5,7,8-tetramethyl- $N$ - $(4,5,6,7-$ tetrachloro-1,3-dioxoisoindolin-2-yl)chroman-2-carboxamide (5k)

Light yellow solid; IR ( $\left.\mathrm{KBr}, \mathrm{v}, \mathrm{cm}^{-1}\right)$ : 3397 (>NH stretch), 2937 (aliphatic C-H), 1705 (>CO stretch); ${ }^{1} \mathrm{H}$ NMR (400 MHz, DMSO-d, ppm): 10.53 (s, -CONH-), $3.49\left(\mathrm{~s}, 3 \mathrm{H},-\mathrm{OCH}_{3}\right), 2.63-2.49\left(\mathrm{~m}, 2 \mathrm{H},>\mathrm{CH}_{2}\right), 2.31-$ $2.25\left(\mathrm{~m}, 1 \mathrm{H},>\mathrm{CH}_{2}\right), 2.07\left(\mathrm{~s}, 6 \mathrm{H},-\mathrm{CH}_{3}\right), 2.03(\mathrm{~s}, 3 \mathrm{H}$, $\left.-\mathrm{CH}_{3}\right), 1.85-1.76\left(\mathrm{~m}, 1 \mathrm{H},>\mathrm{CH}_{2}\right), 1.50\left(\mathrm{~s}, 3 \mathrm{H},-\mathrm{CH}_{3}\right) ;{ }^{13} \mathrm{C}$ NMR (100 MHz, DMSO-d $)$ : 173.2 (-CONH-), 161.2 $(>\mathrm{C}=0,>\mathrm{C}=0), 150.2,146.9,139.9,129.5,127.8,126.4$, $125.7,122.8,118.2,78.3(>\mathrm{C}<), 60.3\left(-\mathrm{OCH}_{3}\right), 30.2$ $\left(>\mathrm{CH}_{2}\right), 24.6\left(-\mathrm{CH}_{3}\right), 20.1\left(>\mathrm{CH}_{2}\right), 12.9\left(-\mathrm{CH}_{3}\right), 12.6$ $\left(-\mathrm{CH}_{3}\right), 12.0\left(-\mathrm{CH}_{3}\right)$; HR-MS: $545.0209(\mathrm{M}+\mathrm{H})^{+}$, calcd. 545.0209 .

(1) 6-methoxy-2,5,7,8-tetramethyl- $N-(4,5,6,7-$ tetrabromo-1,3-dioxoisoindolin-2-yl)chroman-2-carboxamide (51)

White solid; IR $\left(\mathrm{KBr}, \mathrm{v}, \mathrm{cm}^{-1}\right): 3384$ (>NH stretch), 2932 (aliphatic C-H), 1709 (>CO stretch); ${ }^{1} \mathrm{H}$ NMR (400 MHz, DMSO-d, , ppm): 10.49 (s, -CONH-), 3.49 $\left(\mathrm{s}, 3 \mathrm{H},-\mathrm{OCH}_{3}\right), 2.63-2.49\left(\mathrm{~m}, 2 \mathrm{H},>\mathrm{CH}_{2}\right), 2.31-2.25$ $\left(\mathrm{m}, 1 \mathrm{H},>\mathrm{CH}_{2}\right), 2.07\left(\mathrm{~s}, 6 \mathrm{H},-\mathrm{CH}_{3}\right), 2.03\left(\mathrm{~s}, 3 \mathrm{H},-\mathrm{CH}_{3}\right)$, 1.83-1.76 (m, $\left.1 \mathrm{H},>\mathrm{CH}_{2}\right), 1.50\left(\mathrm{~s}, 3 \mathrm{H},-\mathrm{CH}_{3}\right) ;{ }^{13} \mathrm{C}$ NMR (100 MHz, DMSO-d):173.2 (-CONH-), 161.6 $(>\mathrm{C}=0,>\mathrm{C}=0), 150.2,146.9,138.2,129.2,127.8,125.7$, $122.8,121.9,118.2,78.3(>\mathrm{C}<), 60.3\left(-\mathrm{OCH}_{3}\right), 30.2$ $\left(>\mathrm{CH}_{2}\right), 24.6\left(-\mathrm{CH}_{3}\right), 20.1\left(>\mathrm{CH}_{2}\right), 12.9\left(-\mathrm{CH}_{3}\right), 12.6$ $\left(-\mathrm{CH}_{3}\right), 12.0\left(-\mathrm{CH}_{3}\right)$. HR-MS: $718.8027(\mathrm{M}+\mathrm{H})^{+}$, calcd. 718.8025 .

(m) $\quad N$-(1,3-dioxo-1H-pyrrolo [3,4-c]pyridin2(3H)-yl)-6-methoxy-2,5,7,8-tetramethylchroman-2-carboxamide $(5 \mathrm{~m})$

Off-white solid; IR ( $\left.\mathrm{KBr}, \mathrm{v}, \mathrm{cm}^{-1}\right): 3395$ (>NH stretch), 2934 (aliphatic C-H), 1706 (>CO stretch); ${ }^{1} \mathrm{H}$ NMR (400 MHz, DMSO-d, ppm): 10.46 (s, -CONH-), 9.19$9.16(\mathrm{~m}, 2 \mathrm{H}), 7.96-7.95(\mathrm{~m}, 2 \mathrm{H}), 3.49\left(\mathrm{~s}, 3 \mathrm{H},-\mathrm{OCH}_{3}\right)$, 2.64-2.54 (m, $\left.2 \mathrm{H},>\mathrm{CH}_{2}\right), 2.31-2.25\left(\mathrm{~m}, 1 \mathrm{H},>\mathrm{CH}_{2}\right)$, 2.08 (s, 3H, $\left.-\mathrm{CH}_{3}\right), 2.07$ (s, 3H, $\left.-\mathrm{CH}_{3}\right), 2.04$ (s, 3H, $\left.-\mathrm{CH}_{3}\right)$, 
1.84-1.78 (m, $\left.1 \mathrm{H},>\mathrm{CH}_{2}\right), 1.50$ (s, 3H, $\left.-\mathrm{CH}_{3}\right)$; HR-MS: $410.1715(\mathrm{M}+\mathrm{H})^{+}$, calcd. 410.1715 .

(n) $\quad N$-(2,5-dioxopyrrolidin-1-yl)-6-methoxy2,5,7,8-tetramethylchroman-2-carboxamide (5n)

White solid; IR ( $\left.\mathrm{KBr}, v, \mathrm{~cm}^{-1}\right)$ : 3370 (>NH stretch), 2938 (aliphatic C-H), 1701 (>CO stretch); ${ }^{1} \mathrm{H}$ NMR (400 MHz, $\left.\mathrm{CDCl}_{3}, \mathrm{ppm}\right): 8.07$ (s, -CONH-), 3.61 $\left(\mathrm{s}, 3 \mathrm{H},-\mathrm{OCH}_{3}\right), 2.79\left(\mathrm{~s}, 4 \mathrm{H},-\mathrm{CH}_{3}\right), 2.68-2.63(\mathrm{~m}, 2 \mathrm{H}$, $\left.>\mathrm{CH}_{2}\right), 2.38-2.31\left(\mathrm{~m}, 1 \mathrm{H},>\mathrm{CH}_{2}\right), 2.18\left(\mathrm{~s}, 3 \mathrm{H},-\mathrm{CH}_{3}\right)$, $2.15\left(\mathrm{~s}, 3 \mathrm{H},-\mathrm{CH}_{3}\right), 2.14\left(\mathrm{~s}, 3 \mathrm{H},-\mathrm{CH}_{3}\right), 1.98-1.91(\mathrm{~m}, 1 \mathrm{H}$, $\left.>\mathrm{CH}_{2}\right), 1.60$ (s, 3H, $\left.-\mathrm{CH}_{3}\right)$; HR-MS: $361.4043(\mathrm{M}+\mathrm{H})^{+}$, calcd. 361.1764 .

(o) 6-(benzyloxy)-N-(1,3-dioxoisoindolin2-yl)-2,5,7,8-tetramethylchroman-2-carboxamide (5o)

White solid;IR $\left(\mathrm{KBr}, \mathrm{v}, \mathrm{cm}^{-1}\right): 3400$ (>NH stretch), 2925 (aliphatic C-H), 1731 (>CO stretch) ; ${ }^{1} \mathrm{H}$ NMR (400 MHz, DMSO-d $\left.{ }_{6}, \mathrm{ppm}\right): 10.35$ (s, -CONH-), 7.94$7.88(\mathrm{~m}, 4 \mathrm{H}), 7.45$ (d, J=7.0 Hz, 2H), 7.38 (t, J=8.0 Hz, $2 \mathrm{H}), 7.34-7.30(\mathrm{~m}, 1 \mathrm{H}), 4.60(\mathrm{~s}, 2 \mathrm{H}), 2.65-2.52(\mathrm{~m}, 2 \mathrm{H}$, $\left.>\mathrm{CH}_{2}\right), 2.31-2.25\left(\mathrm{~m}, 1 \mathrm{H},>\mathrm{CH}_{2}\right), 2.12\left(\mathrm{~s}, 3 \mathrm{H},-\mathrm{CH}_{3}\right)$, $2.10\left(\mathrm{~s}, 3 \mathrm{H},-\mathrm{CH}_{3}\right), 2.09\left(\mathrm{~s}, 3 \mathrm{H},-\mathrm{CH}_{3}\right), 1.86-1.77(\mathrm{~m}$, $\left.1 \mathrm{H},>\mathrm{CH}_{2}\right), 1.51\left(\mathrm{~s}, 3 \mathrm{H},-\mathrm{CH}_{3}\right) ;{ }^{13} \mathrm{C} \mathrm{NMR}(100 \mathrm{MHz}$, DMSO-d $)_{6}: 173.3$ (-CONH-), $165.5(>\mathrm{C}=0,>\mathrm{C}=0)$, 149.0, 147.1, 138.1, 135.9, 129.8, 128.8, 128.4, 128.1, 126.0, 124.3, 122.9, 118.4, $78.3(>\mathrm{C}<), 74.7\left(>\mathrm{CH}_{2}\right)$, $30.2\left(>\mathrm{CH}_{2}\right), 24.6\left(>\mathrm{CH}_{3}\right), 20.1\left(>\mathrm{CH}_{2}\right), 13.2\left(>\mathrm{CH}_{3}\right)$, $12.7\left(>\mathrm{CH}_{3}\right), 12.3\left(>\mathrm{CH}_{3}\right)$; HR-MS: $507.1896(\mathrm{M}+\mathrm{Na})^{+}$, calcd. 507.1916.

(p) 6-(benzyloxy)-2,5,7,8-tetramethyl- $N$ (4,5,6,7-tetrachloro-1,3-dioxoisoindolin2-yl)chroman-2-carboxamide (5p)

White solid; IR ( $\left.\mathrm{KBr}, \mathrm{v}, \mathrm{cm}^{-1}\right): 3414$ (>NH stretch), 2931 (aliphatic C-H), 1739 (>CO stretch); ${ }^{1} \mathrm{H}$ NMR (400 MHz, DMSO-d, ppm): 10.57 (s, -CONH-), 7.46 (d, J=7.1 Hz, 2H), 7.38 (t, J=7.0 Hz, J=7.8 Hz, 3H), 7.33-7.30 (m, 1H), $4.60(\mathrm{~s}, 2 \mathrm{H}), 2.65-2.50(\mathrm{~m}, 2 \mathrm{H}$, $\left.>\mathrm{CH}_{2}\right), 2.34-2.30\left(\mathrm{~m}, 1 \mathrm{H},>\mathrm{CH}_{2}\right), 2.12\left(\mathrm{~s}, 3 \mathrm{H},-\mathrm{CH}_{3}\right)$, $2.10\left(\mathrm{~m}, 3 \mathrm{H},-\mathrm{CH}_{3}\right), 2.08\left(\mathrm{~s}, 3 \mathrm{H},-\mathrm{CH}_{3}\right), 1.84-1.79(\mathrm{~m}$, $\left.1 \mathrm{H},>\mathrm{CH}_{2}\right), 1.52\left(\mathrm{~s}, 3 \mathrm{H},-\mathrm{CH}_{3}\right) ;{ }^{13} \mathrm{C}$ NMR $(125 \mathrm{MHz}$, DMSO-d $)_{6}: 173.2$ (-CONH-), $161.2(>\mathrm{C}=0,>\mathrm{C}=0)$, 149.0, 147.1, 139.9, 138.1, 129.5, 128.9, 128.4, 128.1, $126.4,126.0,122.8,118.3,78.3(>\mathrm{C}<), 74.7\left(>\mathrm{CH}_{2}\right)$, $30.2\left(>\mathrm{CH}_{2}\right), 24.6\left(>\mathrm{CH}_{3}\right), 20.1\left(>\mathrm{CH}_{2}\right), 13.2\left(>\mathrm{CH}_{3}\right)$, $12.7\left(>\mathrm{CH}_{3}\right), 12.3\left(>\mathrm{CH}_{3}\right)$; HR-MS: $619.0361(\mathrm{M}-\mathrm{H})^{+}$, calcd. 619.0361.

(q) 6-(benzyloxy)-2,5,7,8-tetramethyl- $N$ (4,5,6,7-tetrabromo-1,3-dioxoisoindolin2-yl)chroman-2-carboxamide (5q)
White solid; IR ( $\left.\mathrm{KBr}, \mathrm{v}, \mathrm{cm}^{-1}\right): 3409$ (>NH stretch), 2928 (aliphatic C-H), 1728 (>CO stretch); ${ }^{1} \mathrm{H}$ NMR (400 MHz, DMSO-d, ppm): 10.52 (s, -CONH-), 7.46 (d, J=7.0 Hz, 2H), 7.38 (t, J=7.2 Hz, 2H), 7.34-7.30 $(\mathrm{m}, 1 \mathrm{H}), 4.60$ (s, $2 \mathrm{H}), 2.65-2.50\left(\mathrm{~m}, 2 \mathrm{H},>\mathrm{CH}_{2}\right), 2.34-$ $2.28\left(\mathrm{~m}, 1 \mathrm{H},>\mathrm{CH}_{2}\right), 2.12\left(\mathrm{~s}, 3 \mathrm{H},-\mathrm{CH}_{3}\right), 2.10(\mathrm{~s}, 3 \mathrm{H}$, $\left.-\mathrm{CH}_{3}\right), 2.09\left(\mathrm{~s}, 3 \mathrm{H},-\mathrm{CH}_{3}\right) 1.86-1.78\left(\mathrm{~m}, 1 \mathrm{H},>\mathrm{CH}_{2}\right), 1.52$ (s, 3H, $\left.-\mathrm{CH}_{3}\right) ;{ }^{13} \mathrm{C}$ NMR $(125 \mathrm{MHz}$, DMSO-d $): 173.1$ (-CONH-), 161.6 ( $>\mathrm{C}=0,>\mathrm{C}=0), 149.0,147.1,149.0$, 47.1, 138.2, 138.1, 129.2, 128.9, 128.4, 128.1, 126.0, $122.8,121.9,118.3,78.3(>\mathrm{C}<), 74.7\left(>\mathrm{CH}_{2}\right), 30.2$ $\left(>\mathrm{CH}_{2}\right), 24.7\left(>\mathrm{CH}_{3}\right), 20.1\left(>\mathrm{CH}_{2}\right), 13.2\left(>\mathrm{CH}_{3}\right), 12.7$ $\left(>\mathrm{CH}_{3}\right), 12.3\left(>\mathrm{CH}_{3}\right)$; HR-MS: $794.8340(\mathrm{M}-\mathrm{H})^{+}$, calcd. 794.8341.

(r) 6 - (benzyloxy) $-N-(1,3-$ dioxo-1 H pyrrolo[3,4-c]pyridin-2(3H)-yl)-2,5,7,8-tetramethylchroman-2-carboxamide $(5 \mathbf{r})$

White solid; IR ( $\left.\mathrm{KBr}, \mathrm{v}, \mathrm{cm}^{-1}\right): 3375$ (>NH stretch), 2926 (aliphatic C-H), 1736 (>CO stretch); ${ }^{1} \mathrm{H}$ NMR (400 MHz, DMSO-d, $\mathrm{ppm}$ ): 10.49 (s, -CONH-), 9.20 (s, 1H), 9.17 (d, J=4.6 Hz, 1H), $7.96(\mathrm{~d}, J=5.0 \mathrm{~Hz}, 1 \mathrm{H})$, $7.46(\mathrm{~d}, J=6.8 \mathrm{~Hz}, 2 \mathrm{H}), 7.38(\mathrm{t}, J=6.8 \mathrm{~Hz}, 2 \mathrm{H}), 7.33-$ $7.30(\mathrm{~m}, 1 \mathrm{H}), 4.60(\mathrm{~s}, 2 \mathrm{H}), 2.65-2.49\left(\mathrm{~m}, 2 \mathrm{H},>\mathrm{CH}_{2}\right)$, 2.33-2.27 (m, $\left.1 \mathrm{H},>\mathrm{CH}_{2}\right), 2.12\left(\mathrm{~s}, 3 \mathrm{H},-\mathrm{CH}_{3}\right), 2.10$ $\left(\mathrm{s}, 3 \mathrm{H},-\mathrm{CH}_{3}\right), 2.09$ (s, $\left.3 \mathrm{H},-\mathrm{CH}_{3}\right), 1.86-1.80(\mathrm{~m}, 1 \mathrm{H}$, $\left.>\mathrm{CH}_{2}\right), 1.52\left(\mathrm{~s}, 3 \mathrm{H},-\mathrm{CH}_{3}\right)$; HR-MS: $508.1848(\mathrm{M}+\mathrm{Na})^{+}$, calcd. 508.1848 .

(s) 6-(benzyloxy)- $N$-(2,5-dioxopyrrolidin1-yl)-2,5,7,8-tetramethylchroman-2-carboxamide (5s)

White solid; IR ( $\left.\mathrm{KBr}, \mathrm{v}, \mathrm{cm}^{-1}\right): 3370$ (>NH stretch), 2927 (aliphatic C-H), 1732 (>CO stretch); ${ }^{1} \mathrm{H}$ NMR (400 $\left.\mathrm{MHz}, \mathrm{CDCl}_{3}, \mathrm{ppm}\right): 8.10$ (s, -CONH-), 7.48 (d, J=7.4 Hz, 2H), 7.40 (t, J=7.0 Hz, 2H), 7.35-7.31 $(\mathrm{m}, 1 \mathrm{H}), 4.68(\mathrm{~s}, 2 \mathrm{H}), 2.79\left(\mathrm{~s}, 4 \mathrm{H},>\mathrm{CH}_{2}\right), 2.70-2.63$ $\left(\mathrm{m}, 2 \mathrm{H},>\mathrm{CH}_{2}\right), 2.39-2.29\left(\mathrm{~m}, 1 \mathrm{H},>\mathrm{CH}_{2}\right), 2.21(\mathrm{~s}, 3 \mathrm{H}$, $\left.-\mathrm{CH}_{3}\right), 2.17\left(\mathrm{~s}, 3 \mathrm{H},-\mathrm{CH}_{3}\right), 2.15\left(\mathrm{~s}, 3 \mathrm{H},-\mathrm{CH}_{3}\right), 2.02-1.93$ (m, $\left.1 \mathrm{H},>\mathrm{CH}_{2}\right), 1.61$ (s, 3H, $-\mathrm{CH}_{3}$ ); HR-MS: 459.1896 $(\mathrm{M}+\mathrm{Na})^{+}$, calcd. 459.1894 .

(t) 6-(benzyloxy)-2,5,7,8-tetramethyl- $N$ (3-methyl-2,5-dioxo-2,5-dihydro-1 $H$-pyrrol1-yl)chroman-2-carboxamide (5t)

Light yellow solid;IR (KBr, v, cm $\left.{ }^{-1}\right): 3375$ (>NH stretch), 2926 (aliphatic C-H), 1736 (>CO stretch); ${ }^{1} \mathrm{H}$ NMR (400 MHz, $\left.\mathrm{CDCl}_{3}, \mathrm{ppm}\right): 8.02$ (s, -CONH-), 7.48 (d, J=7.0 Hz, 2H), $7.40(\mathrm{t}, J=7.0 \mathrm{~Hz}, 2 \mathrm{H}), 7.35-7.32$ $(\mathrm{m}, 1 \mathrm{H}), 6.42(\mathrm{~d}, J=1.8 \mathrm{~Hz}, 1 \mathrm{H}), 4.70(\mathrm{~s}, 2 \mathrm{H}), 2.66$ $\left(\mathrm{t}, J=6.6 \mathrm{H} \approx, 2 \mathrm{H},>\mathrm{CH}_{2}\right), 2.39-2.33\left(\mathrm{~m}, 1 \mathrm{H},>\mathrm{CH}_{2}\right), 2.22$ $\left(\mathrm{s}, 3 \mathrm{H},-\mathrm{CH}_{3}\right), 2.18\left(\mathrm{~s}, 3 \mathrm{H},-\mathrm{CH}_{3}\right), 2.17\left(\mathrm{~s}, 3 \mathrm{H},-\mathrm{CH}_{3}\right)$, $2.11\left(\mathrm{~d}, \mathrm{~J}=1.8 \mathrm{H}_{2}, 3 \mathrm{H},-\mathrm{CH}_{3}\right), 1.98-1.91\left(\mathrm{~m}, 1 \mathrm{H},>\mathrm{CH}_{2}\right)$, 1.61 (s, $\left.3 \mathrm{H},-\mathrm{CH}_{3}\right) ;{ }^{13} \mathrm{C}$ NMR (100 MHz, $\left.\mathrm{CDCl}_{3}\right): 173.4$ 
(-CONH-), $168.4(>\mathrm{C}=0), 167.1$ ( $>\mathrm{C}=0), 149.4,146.1$, $145.3(>\mathrm{C}<), 137.7,128.8,128.6,128.0,127.8,126.9$, 122.5, 118.4, $79.0(>\mathrm{C}<), 74.8\left(>\mathrm{CH}_{2}\right), 29.9\left(>\mathrm{CH}_{2}\right)$, $23.9\left(-\mathrm{CH}_{3}\right), 20.1\left(-\mathrm{CH}_{3}\right), 13.0\left(>\mathrm{CH}_{2}\right), 12.3\left(-\mathrm{CH}_{3}\right), 12.1$ $\left(-\mathrm{CH}_{3}\right), 11.6\left(-\mathrm{CH}_{3}\right)$; HR-MS: $449.2076(\mathrm{M}+\mathrm{H})^{+}$, calcd. 449.2080.

\section{Antiepileptic Activity}

The antiepileptic evaluation of the synthesized compounds was performed using reported procedures. ${ }^{23,24}$ Male albino mice (CF-1 strain, 18-25 g) were used. Test compounds were dissolved in polyethyleneglycol (PEG-400) and were administered intraperitoneally (i.p.). The MES convulsion was measured using Ugo Basile Convulsiometer. Sodium valproate and phenytoin were used as standards in antiepileptic tests.

\section{Neurotoxicity screening}

Neurotoxicity induced by compounds (5a-t) was measured in mice by the rotarod test. ${ }^{21}$ The mice $(n=6)$ were trained to stay on rotating rotarod $(3.2 \mathrm{~cm}$ diameter) that rotated at six revolutions per minute. Trained mice were given IP injection of compounds at doses (30 mg/kg, $100 \mathrm{mg} / \mathrm{kg}$ and $300 \mathrm{mg} / \mathrm{kg}$ ). After $30 \mathrm{~min}$ the mice were allowed to place on the rotating rod. The mice fail to manage equilibrium on rod for $1 \mathrm{~min}$ were noted for neurotoxicity. ${ }^{25}$

\section{Pentylenetetrazole seizure (PTZ) method}

The mice $(n=6)$ were administered by compounds intraperitonealy. After $30 \mathrm{~min}$, mice were treated with subcutaneous injection of pentylenetetrazole (PTZ, $60 \mathrm{mg} / \mathrm{kg}$ ). Then mice were placed singly in isolatedplastic cagesand were observed for $60 \mathrm{~min}$. The failure to detect an episode of clonic spasms of at least 5 secis termed as protection. These results were compared with standard drug, sodium valproate $(200 \mathrm{mg} / \mathrm{kg}$, i.p).

\section{Maximal electroshock seizure (MES) method}

Antiepileptic activity of compounds (5a-t) was measured by maximal electroshock seizure (MES) method. ${ }^{23}$ Albino mice were divided into group of 6 animals each. Maximal electroshock seizure were elicited with a $60 \mathrm{~Hz}(50 \mathrm{~mA})$ for $0.2 \mathrm{sec}$ via corneal electrodes. Compounds were intraperitonealy injected $(0.01 \mathrm{~mL} / \mathrm{kg}$ body weight) at $30 \mathrm{mg} / \mathrm{kg}, 100 \mathrm{mg} / \mathrm{kg}$ and $300 \mathrm{mg} /$ $\mathrm{kg}$ doses and activity was evaluated after $0.5 \mathrm{hr}$ and 4 hr. Abolishment of the hind limb tonic extensor spasm was noted.

\section{CONCLUSION}

The success of the proposed scheme is depicted as a series of chromanan hydride analogs (5a-t) was synthesized in good yield and purity by the condensation reaction of chroman hydrazides and different anhydrides. The antiepileptic activity of these analogs was determined by PTZ and MES and compared with standard drugs, sodium vaproate and phenytoin, respectively. All compounds were found to be active by applying PTZ method which supports the inhibition of PTZinduced seizure in mice by enhancing GABAergic neurotransmission. Whereas by MES method some, not all, of the compounds were found to have good activity. At low dose of $30 \mathrm{mg} / \mathrm{kg}$, compound $5 \mathrm{j}$ possessed similar or better activity as compare to standard drugs and considered as the most potent antiepileptic among the series. Most of the compounds of the series displayed lack of neurotoxicity except for compounds $5 \mathrm{~m}$ and $5 \mathrm{p}$ which were toxic at the dose of $300 \mathrm{mg} / \mathrm{kg}$ after4 $\mathrm{h}$. Thus, a platform for further development of related compounds into promising antiepileptic agents is suggested with lesser neurotoxicity.

\section{ACKNOWLEDGEMENT}

Pinki Rawat is thankful to UGC-RGNF for providing National Doctoral Fellowship and contingency grant as financial support.

\section{CONFLICT OF INTEREST}

The authors declare no conflict of interest.

\section{ABBREVIATIONS}

PTZ: Pentylenetetrazole Seizure; MES: Maximal electroshock seizure; NT: Neurological toxicity; AEDs: Antiepileptic Drugs; SAR: Structure Activity Relationship; FT-IR: Fourier-transform infrared spectroscopy; ${ }^{1} \mathbf{H}$-NMR: Proton nuclear magnetic resonance; ${ }^{13} \mathbf{C}-$ NMR: Carbon-13 nuclear magnetic resonance; GABA: Gamma aminobutyric acid; TMS: Tertramethyl Silane; IP: Intraperitoneal.

\section{REFERENCES}

1. Aragade P, Kolhe S, Kamble H, Baheti D, Maddi V. Synthesis and Preliminary Evaluation of Some Substituted Pyrazoles as Anticonvulsant Agents. Int J Drug Des Discovery. 2012;3(1):688-93.

2. Bell G, Sander J. The epidemiology of epilepsy: The size of the problem. Seizure. 2002;11(Suppl A):306-14.

3. Lagae L. Cognitive side effects of anti-epileptic drugs: The relevance in childhood epilepsy. Seizure. 2006;15(4):235-41.

4. Picot MC, Moulinier MB, Daurs JP, Dujols P, Crespel A. The prevalence of epilepsy and pharmacoresistant epilepsy in adults: A population-based study in a western European country. Epilepsia. 2008;49(7):1230-8. 
5. Bhat M, Siddiqui N, Khan S. Synthesis of novel 3-(4-acetyl-5h/methyl-5substitutedPhenyl-4,5-dihydro-1,3,4-oxadiazol-2-yl)-2h-chromen-2-ones as potential anti-convulsant agents. Acta Pol Pharm. 2008;65(2):235-9.

6. Eddy CM, Rickards HE, Cavanna AE. The cognitive impact of antiepileptic drugs. Ther Adv Neurol Disord. 2011;4(6):385-407.

7. Emami S, Kebriaeezadeh A, Ahangar N, Khorasani R. Imidazolylchromanoneoxime ethers as potential anticonvulsant agents: Anticonvulsive evaluation in PTZ-kindling model of epilepsy and SAR study. Bioorg Med Chem Lett. 2011;21(2):655-9.

8. Semwal A. Novel B, B -Diphenyl propionic acid Amide derivatives showing anti-inflammatory and anticonvulsant activity. Int $\mathrm{J}$ Med Sci Cli Invent. 2014;1:15-24.

9. Kulandasamy R, Adhikari AV, Stables JP. Design and synthesis of new Amides and Thioamides derived from 3,4-ethylenedioxythiophene as Potential Anticonvulsants. Bull Korean Chem Soc. 2010;31(11):3318-26.

10. Malik S, Ahuja P, Sahu K, Khan SA. Design and synthesis of new of 3-(benzo[d]isoxazol-3-yl)-1-substituted pyrrolidine-2, 5-dione derivatives as anticonvulsants. Eur J Med Chem. 2014;84:42-50.

11. Rawat P, Verma SM. Design and synthesis of Chroman derivatives with dual Anti-breast cancer and antiepileptic activities. Drug Des Dev Ther. 2016;10:2779-88.

12. Rawat $\mathrm{P}$, Verma SM. Docking studies of substituted chroman analogs at estrogen receptor. Asian J Pharm Clin Res. 2015;8:88-92.

13. Florence X, Dilly S, Tullio PD, Pirotte B, Lebrun P. Modulation of the 6-position of benzopyran derivatives and inhibitory effects on the insulin releasing process. Bioorg Med Chem. 2011;19(13):3919-28.

14. Conti C, Monaco LP, Desideri N. Design, synthesis and in vitro evaluation of novel chroman-4-one, chroman and $2 \mathrm{H}$-chromene derivatives as human rhinovirus capsid-binding inhibitors. Bioorg Med Chem. 2011;19(24):7357-64.

15. Kraus GA, Mengwasser J, Maury W, Oh C. Synthesis of chroman aldehydes that inhibit HIV. Bioorg Med Chem Lett. 2011;21(5):1399-401.
16. Rawat $P$, Verma SM. Synthesis and pharmacological evaluation of 6-hydroxy2,5,7,8-tetramethyl-N'-(2-oxoindolin-3-ylidene)chroman-2-carbohydrazide derivatives asantimicrobial agents. J Chem Pharm Res. 2016;8(3):149-54.

17. Hyatt JA. Convenient Preparation of 2,7,8-Trimethyl-6-hydroxychroman-2carboxylic Acid ( $\gamma$-Trolox). Synth Comm. 2008;38(1):8-14.

18. Koufaki M, Koufaki M, Theodorou E, Galaris D, Nousis L, Katsanou ES, et al. Chroman/Catechol Hybrids: Synthesis and Evaluation of Their Activity against Oxidative Stress Induced Cellular Damage. J Med Chem. 2006;49(1):300-6.

19. Chen W, Park SK, Yu W, Xiong A, Sanders BG, Kline K. Synthesis and screening of novel Vitamin $E$ derivatives for anticancer functions. Eur J Med Chem. 2012;58:72-83.

20. Lopez GV, Blanco F, Hernandez P, et al. Second generation of a-tocopherol analogs-nitric oxide donors: Synthesis, physicochemical and biological characterization. Bioorg Med Chem. 2007;15(18):6262-72.

21. Dunham NW, Miya TS. A note on a simple apparatus for detecting neurological deficit in rats and mice. J Am Pharm Assoc Am Pharm Assoc. 1957;46(3):2089.

22. Fang $Y$, Sun C, Liu D, Wang S, Quan Z. Synthesis and anticonvulsant activity evaluation of 3-alkoxy-4-(4-(hexyloxy/heptyloxy)phenyl)-4H-1,2,4-triazole. Iran J Pharm Res. 2015;14(1):77-87.

23. Krall RL, Penry JK, White BG, Kupferberg HJ, Swinyard EA. Antiepileptic drug development: II. Anticonvulsant drug screening. Epilepsia.1978;19(4):409-28.

24. Porter RJ, Cereghino JJ, Gladding GD, et al. Antiepileptic drug development program. Cleveland Clin Quart. 1984;51(2):293-305.

25. Kumar R, Singh T, Singh H, Jain S, Roy RK. Design, synthesis and anticonvulsant activity of some New 6,8-halo-substituted-2h-[1,2,4] triazino[5,6-b]indole-3(5h)-one/-thione and 6,8-halo-substituted 5-methyl2h-[1,2,4]triazino[5,6-b]indol-3(5h)-one/-thione. EXCLI Journal. 2014;13:225-40.

\section{PICTORIAL ABSTRACT}

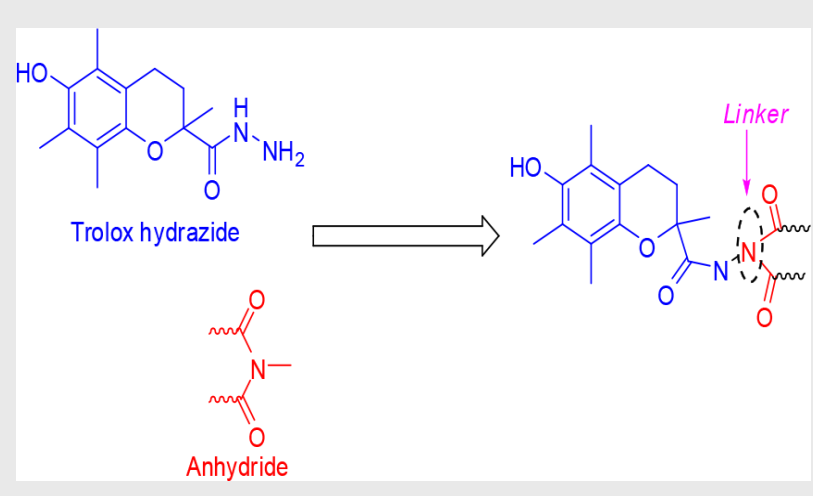

\section{SUMMARY}

Chromans are considered to be an important chemical synthon, associated with a broad range of biological effects including antiepileptic activity. Antiepileptic study of test compounds (5a-t) in male albino mice by Maximal electroshock seizure (MES) and pentylenetrazole induced convulsion (PTZ) methods was carried out. Compound $5 \mathrm{j}(30 \mathrm{mg} / \mathrm{kg})$ showed highest and advanced antiepileptic activity than reference drugs in both the methods. Moreover, none of the compounds showed neurotoxicity at $30 \mathrm{mg} / \mathrm{kg}$ and $100 \mathrm{mg} / \mathrm{kg}$, as determined by the rotarod test.

\section{About Authors}

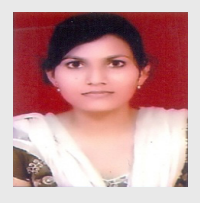

Dr. Pinki Rawat, has completed her Ph.D from Birla Institute of Technology, Ranchi, India. Her research interest are in the area of synthesis of heterocyclic compounds, molecular docking, QSAR, antiepileptic, anticancer, antimicrobial and antioxidant. 
Dr. Saurabh Manaswita Verma, is currently working at Birla Institute of Technology, Ranchi. Her research interest are in the area of docking, Synthetic Chemisty, Natural Product Chemistry and Computer Aided Drug Design.

Mr. Piyush Kumar, is Persuing his Ph.D from Integral University, Lucknow, India. He has done MS Pharm from NIPER, Raebareli, India. His research interest in the area of organic chemistry, medicinal chemistry and nanotechnology.

Cite this article: Rawat P, Manaswitaverma S, Kumar P. Novel Chroman Analogs as Promising Heterocyclic Compounds: Their Synthesis and Antiepileptic Activity. Indian J of Pharmaceutical Education and Research. 2019;53(4s):s655-s665. 